\title{
Update on adolescent mental health
}

\author{
S Paruk, MB ChB, FCPsych (SA), Child Psych (SA), MMed; E Karim, MB ChB, FCPsych (SA), Child Psych (SA) \\ Discipline of Psychiatry, School of Clinical Medicine, College of Health Sciences, University of KwaZulu-Natal, Durban; and \\ King Dinizulu Hospital, Durban, South Africa
}

Corresponding author: S Paruk (paruks4@ukzn.ac.za)

Adolescence is a period of significant physical, social and emotional change and therefore a vulnerable period for the development of mental illness. Many psychiatric disorders have their onset during adolescence. Approximately $20 \%$ of children have a mental health disorder, but the majority of disorders are not detected and treated. Risk factors for mental illness include: genetic vulnerability, neurobiological factors and psychosocial stressors. Common mental disorders during this period are mood, anxiety and substance related, with adolescents often presenting with comorbidity. Healthcare practitioners should screen for adolescent mental illness and associated suicide risk. Brief screening tools such as the Strengths and Difficulties Questionnaire are useful in detecting mood and anxiety disorders in primary care settings. While there is increasing evidence for the efficacy of psychotropic medications in adolescents, more research is still required. Management of adolescent mental health problems generally requires a combination of psychotropic medication and psychosocial interventions. Early intervention and support in an integrated medical and psychiatric healthcare system is required.

S Afr Med J 2016;106(6):548-550. DOI:10.7196/SAMJ.2016.v106i6.10943

\section{Scope of mental health problems in adolescence}

It is estimated that about $20 \%$ of children and adolescents have a mental health disorder and approximately half of all mental illness and substance-related problems start at the age of 14 years. ${ }^{[1]}$ The risk of mental health problems is further exacerbated in vulnerable environments with poor social support and socioeconomic inequalities, such as developing countries. ${ }^{[2]}$ In a study of mental health among 15 - 19-year-old youth in five cities globally, female adolescents from Johannesburg reported the highest levels of depression and post-traumatic stress symptoms (44.6\% and $67.0 \%$, respectively). ${ }^{[2]}$ Suicide remains the second leading cause of death among young people worldwide ${ }^{[3]}$ Common psychiatric disorders in adolescents, including anxiety-, mood-, trauma- and stressorrelated disorders, are all associated with increased suicide risk. ${ }^{[3]}$

\section{Importance of assessing and treating} mental health problems in adolescence The majority of adolescents with mental illness remain undiagnosed and untreated, and services are often fragmented. ${ }^{[4]}$ Mental illness in adolescents is associated with significant disease burden, such as poor academic achievement, suicide, violence, substance use, pregnancy and potentially increased risk of psychopathology later in adulthood. ${ }^{[4]}$ The aetiology of mental illness in adolescents is multifactorial and includes biological factors, such as genetic vulnerability, in utero exposure to toxins, substance abuse and head trauma. Psychosocial factors include abuse, neglect, bereavement, family conflict, bullying and stressful life events. ${ }^{[4]}$ Protective factors include familial ties and social support. ${ }^{[2]}$

While the Diagnostic and Statistical Manual of Mental Disorders, 5th edition (DSM-5) diagnostic criteria are generally the same for adolescents and adults, the psychiatric assessment of the adolescent can be more challenging. ${ }^{[5]}$ Adolescence is a period of significant emotional and behavioural change, which makes distinguishing normal development from psychiatric illness difficult. Most adolescents are not able to independently access health services, and are often accompanied by a caregiver. This may raise challenges, as adolescents may be reluctant to engage with the practitioner, may fear disclosure of personal information to the caregiver, or may fear stigmatisation by others. Practitioners should guard against any perception of collusion with the caregiver.

Adolescents may often present with vague, nonspecific symptoms, as they may be unable to express their emotional distress. Finally, there is often comorbidity between the psychiatric disorders, substance use and medical disorders, which may further contribute to the challenge. ${ }^{[4]}$

The substantial evidence of a high prevalence and burden of mental illness in adolescence suggests a need for greater awareness of psychiatric disorders. Primary healthcare practitioners should consider mental illness and suicide risk when assessing adolescents. A variety of screening tools are available to assist with assessment, including: the Paediatric Symptom Checklist, the Strengths and Difficulties Questionnaire (SDQ), and the Reporting Questionnaire for Children. ${ }^{[6-8]}$ The SDQ has been used in the South African setting and is available in several languages.

Although the evidence base for management of psychiatric disorders in adolescents is improving, there is still a dire need for further pharmacological and psychosocial intervention studies. ${ }^{[4]}$ General treatment principles include establishing a therapeutic alliance, involvement of family and school, and use of a multimodal treatment plan that includes psycho-education and pharmacological and psychosocial interventions. There is increasing recognition of the efficacy of psychotropic medication in this age group. Medication use is determined by the type and severity of the psychiatric disorder, past psychiatric history, family history, medical history and patient and family preference. ${ }^{[9]}$ When prescribing medication, one has to consider the appropriate dosage and slow titration, and avoid polypharmacy.

\section{Identifying common mental health problems}

The objective of this article is to highlight the high prevalence of psychiatric disorders in adolescents and to provide a casebased clinical overview of common mental health disorders and interventions available for adolescents. 


\section{Case report}

A 15-year-old girl presented after a panic attack. The patient and her parents reported that she suffered from irritable mood, withdrawal, tearful bouts, excessive worry, poor concentration at school (poor marks in last school report), loss of weight and disturbed sleep for 4 months. There were no psychotic symptoms and she did not use substances. On further enquiry the patient revealed that she was almost raped while out with friends. Collateral information from the teacher revealed that the patient had always been anxious, especially during public speaking and interacting with others, and that there was deterioration in her academic work more recently.

The assessment of this patient includes a comprehensive history from the patient and her family, collateral information from the school, a physical examination, a mental state examination and any relevant investigations to exclude a medical disorder.

The diagnostic considerations of the abovementioned adolescent should include the disorders listed below.

\section{Mood disorders \\ Depression}

The prevalence of depression ranges from $4 \%$ to $8 \%$ in adolescents. ${ }^{[10]}$

The DSM-5 diagnostic criteria for depression in adolescents and adults are the same. ${ }^{[5]}$ Adolescents are more likely to present with irritable mood, apathy, sadness, low self-esteem, social withdrawal, insomnia and impaired concentration. They often describe themselves as useless or life as boring. The diagnosis of depression may be missed in older adolescents, as they may present with oppositional or antisocial behaviour, use of substances and problems at school. Fifty percent of adolescents with depression have two or more comorbid disorders, including disruptive behaviour disorders and anxiety disorders. ${ }^{[9]}$

A suicide risk assessment must be part of every evaluation. Hospitalisation may be indicated if the patient is actively suicidal or has recent suicidal behaviour. The treatment of depression is multimodal, with a combination of psychotherapy and increasing evidence for the use of antidepressants in this age group.

Psychotherapeutic interventions include psycho-education of the patient and family, supportive therapy and cognitive behavioural therapy. ${ }^{\left[{ }^{[9]}\right.}$ Treatment with antidepressants is indicated for moderate to severe depression, recurrent depression and poor response to psychotherapy. Selective serotonin reuptake inhibitors (SSRIs) are considered as the first-line intervention for adolescents with depression. In this regard, the most evidence has currently been accumulated for the SSRI, fluoxetine. ${ }^{[1]}$ Tricyclic antidepressants are not effective for paediatric depression. The risk of suicide with SSRI antidepressant initiation in children and adolescents is controversial. ${ }^{[1]}$ The potential risk of treatment-emergent agitation and suicidal ideation associated with SSRI medication can be managed with close monitoring of the patient for self-harm and informed consent from the caregiver for the use of psychotropic medication. Treatment with antidepressants should be continued for 6 - 12 months after recovery and should be tapered off over $6-12$ weeks at the end of treatment. ${ }^{[9]}$

\section{Trauma- and stressor-related disorders Post-traumatic stress disorder}

There is a high prevalence of trauma in our society and approximately a quarter of all individuals who experience significant trauma may develop post-traumatic stress disorder (PTSD).$^{[12]}$

Adolescents with PTSD develop symptoms in response to a traumatic event involving actual or threatened death, serious injury or sexual violence. The patient may have experienced the event personally or it may have occurred to another, with the patient witnessing or being informed of it. Patients with this disorder, experience intrusive symptoms such as recurrent recollections, dreams, flashbacks and persistent severe psychological and physiological reactions to reminders of the event. They attempt to avoid thinking about the occurrence and external reminders of the trauma. Disturbances in mood and cognition occur, such as fear, guilt, detachment and memory impairment. Furthermore, there may be alterations in arousal and reactivity levels, such as hypervigilance and increased startle response. ${ }^{[5]}$

\section{Acute stress disorder}

Acute stress disorder (ASD) presents very similarly to PTSD. While the traumatic event criteria and symptoms are identical, it differs with regard to duration. ASD symptoms last for 3 days - 1 month, while PTSD symptoms are present for $>1$ month. ${ }^{\left[{ }^{[j]}\right.}$ It is estimated that half of PTSD patients will first present with ASD. After exposure to a traumatic event, many adolescents experience a degree of distressing symptoms, but will not progress to developing a trauma-related disorder. For those manifesting only mild symptoms for $<4$ weeks, it may be appropriate to adopt a watchful waiting position. ${ }^{[12]}$

The evidence base supports the use of trauma-focused psychotherapy, in particular cognitive behavioural therapy. ${ }^{[12]}$ Medication may be warranted for severe and persisting symptoms, as well as for symptoms that fail to respond to psychotherapy. There is a marked paucity of pharmacological treatment studies in adolescents. Based on the available data, an SSRI is an appropriate choice, noting the abovementioned cautions. Psychotherapy and medication are often combined. ${ }^{[13]}$

\section{Adjustment disorder}

Adolescents may also react to stressors of lesser severity than those associated with the conditions noted above. An adjustment disorder refers to the development of emotional or behavioural symptoms in response to and within 3 months of a stressor that does not meet the criteria for a traumatic event. The reaction is disproportionate to the severity of the stressor and is associated with functional impairment. ${ }^{[5]}$ The disorder may present with varied symptoms, such as anxiety, depression and behavioural disturbances. Adjustment disorder in adolescents is associated with an increased risk of suicidal behaviour and, at a later stage, psychiatric disorders. ${ }^{[14]}$

Crisis intervention and brief psychotherapy may be useful options. Pharmacotherapy is generally not indicated, except for short-term relief of insomnia and anxiety symptoms. ${ }^{[14]}$

\section{Anxiety disorders}

\section{Social anxiety disorder (social phobia)}

Patients with this disorder experience marked fear or anxiety in response to social situations in which they are under the scrutiny of others. They fear that their behaviour will be embarrassing, humiliating or lead to rejection, or be perceived as offensive to others. The severity of symptoms is in excess of the actual risks posed by the situation. Patients avoid such situations or endure them with intense fear or anxiety. ${ }^{[5]}$ While many people experience some degree of anxiety in social situations, it is generally only those with the disorder that experience resultant functional impairment.

\section{Generalised anxiety disorder}

The prevalence of generalised anxiety disorder (GAD) among children and adolescents has been estimated to be as high as $10 \% \cdot{ }^{[15]}$

GAD is characterised by excessive anxiety and worry over a variety of day-to-day issues. The excessive anxiety occurs almost daily and persists for at least 6 months. The symptoms may include 
restlessness, muscle tension, fatiguability, irritability, disturbed concentration and sleep, ${ }^{[5]}$ and panic attacks.

Panic attacks refer to brief periods of intense anxiety. During an attack a multitude of somatic symptoms may occur, such as tachycardia, shortness of breath, nausea and paraesthesia. Psychological symptoms such as fear of dying or losing control, derealisation, and depersonalisation may also occur. ${ }^{[5]}$ Panic attacks frequently occur in association with other psychiatric disorders. For example, a learner with social anxiety disorder may experience a panic attack if required to deliver a speech in front of an audience, or someone with PTSD may have an attack if reminded of their trauma. These panic attacks can be anticipated because they are triggered by identifiable situations.

\section{Panic disorder}

Panic disorder comprises recurrent panic attacks that are unexpected, as they do not occur in response to an identifiable situation. Furthermore, the attacks induce a prolonged period (at least 1 month) of persistent worry or maladaptive behavioural change..$^{[5]}$

As somatic symptoms predominate, it is important to first exclude the possibility that the symptoms are the result of another medical condition or are substance related.

Patients often display symptoms of more than one anxiety disorder. ${ }^{[5]}$ The anxiety disorders share commonalities in aetiology and symptomatology; therefore, similar treatment approaches are used. The established treatments for anxiety disorders are psychotherapy (with a good evidence base for cognitive behavioural therapy), medications (most evidence favouring the SSRIs), and combinations of these approaches. ${ }^{[13]}$ There is only a small role for the use of benzodiazepines owing to side-effects and the risk of addiction.

While adolescents may also suffer from other anxiety disorders, such as separation anxiety disorder, specific phobias and agoraphobia, these are not common in this age group and therefore beyond the scope of this article.

\section{Conclusion}

Psychiatric disorders in adolescents are common and often pose many clinical challenges with regard to assessment and treatment. Mental illness in adolescents is associated with significant short- and long-term morbidity. There is therefore an urgent need to increase awareness, and improve screening, detection and treatment of mental illness in this vulnerable group. An integrated healthcare system that provides medical and mental healthcare at primary and secondary level is critical to address the mental healthcare gap in adolescent services.

\section{References}

1. Kessler RC, Berglund P, Demler O, et al. Lifetime prevalence and age of onset distributions of DSM IV in the National Comorbidity Survey Replication. Arch Gen Psychiatry 2005;62(6):593-602. DOI:10.1001/archpsyc.62.6.593

2. Cheng $Y$, XianChen L, Chaohua L, et al. The association between social support and mental health among the vulnerable adolescents in five cities: Findings from the study of the well-being of adolescents in vulnerable environments. J Adolesc Health 2014;55:S31-S38. DOI:10.1016/ of adolescents in vula
jadohealth.2014.08.020

3. Hawton K, Saunders KEA, O'Conner RC. Self-harm and suicide in adolescents. Lancet 2012;23:2773-2782.

4. Patel V, Flisher AJ, Hetrick S, McGorry P. Mental health of young people: A global public health

challenge. Lancet 2007;369:1302-1313.
5. American Psychiatric Association. Diagnostic and Statistical Manual of Mental Disorders. 5th ed. 5. American Psychiatric Association. Diagnostic and Sta

6. Jellinek MS, Murphy JM. Brief psychosocial screening in outpatient pediatric practice. J Pedriat 1986;109:371-378.

7. Goodman R. The Strengths and Difficulties Questionnaire: A research note. J Child Psychol Psychiatry 1997;38:581-586.

8. Tedesse B, Kabede D, Tegegne T, Alem A. Childhood behavioural disorders in Ambo district, Western Ethiopia. II. Validation of the RQC. Acta Psychiatr Scand Suppl 1999;397:98-101.

. Birmaher B, Ryan ND, Williamson DE, et al. Childhood and adolescent depression: A review of the past 10 years. Part I. J Am Acad Child Adolesc Psychiatry 2003;35:1427-1439.

10. Costello J, Erkanli E, Angold A. Is there an epidemic of child or adolescent depression? J Child Psychol Psychiatry 2006;47:1263-1271.

11. Birmaher B, Brent D; AACAP working group on quality issues. Practice parameters for the assessment and treatment of children and adolescents with depressive disorders. J Am Acad Child Adolesc Psychiatry 2007;46:1503-1526.

12. National Institute for Health and Clinical Excellence. Post-Traumatic Stress Disorder (PTSD): The Management of PTSD in Adults and Children in Primary and Secondary Care. Clinical Guideline 26. London: NICE, 2006.

13. Rynn M, Puliafico A, Heleniak C, et al. Advances in pharmacotherapy for pediatric anxiety disorders Depress Anxiety 2011;28:76-87. DOI:10.1002/da.20769

14. Casey P, Bailey S. Adjustment disorders: The state of the art. World Psychiatry 2011;10:11-18.

15. Keeton CP, Kolos AC, Walkup JT. Pediatric generalized anxiety disorder: Epidemiology, diagnosis, and management. Paediatr Drugs 2009;11(3):171-83. DOI:10.2165/00148581-200911030-00003

\section{Further reading}

World Health Organization. Child and Adolescent Mental Health Resources: Global Concerns and Implications for the Future. Geneva: WHO, 2005. http:www.who.int/mental_health/publications (accessed 26 April 2016). 